\title{
PARTICLE MOTION ON THE SURFACE OF A CONCAVE SOIL-TILLING DISK
}

\author{
Serhit F. Pylypaka ${ }^{a}$, Mykola B. KlendiI ${ }^{b}$, Oleksandra M. KlendiI ${ }^{b, *}$ \\ ${ }^{a}$ National University of Life and Environmental Sciences of Ukraine, Heroiv Oborony Str., 15, Kyiv, Ukraine \\ ${ }^{b}$ Separated Subdivision of National University of Life and Environmental Sciences of Ukraine Berezhany \\ Agrotechnical Institute, Akademichna Str., 20, Berezhany, Ukraine. \\ * corresponding author: klendii_o@ukr.net
}

\begin{abstract}
Relative particle motion on the internal rough surface of a concave soil-tilling disk, which rotates around horizontal axis under a soil reaction force, has been considered. A disk blade is positioned in a vertical plane, which makes an incidence angle with the direction of the machine movement. This angle has its acceptable limit and when it exceeds the limit, it causes disk dragging. In the paper, it has been assumed that dragging was non-existent, the rotational rate of a disk is stable and it depends on stabilized velocity of the machine movement and on an incidence angle. When a machine is operated, soil particles get onto an internal disk surface and perform a relative motion, which determines the slipping trajectory of a particle on a disk surface. The trajectory of the absolute particle motion relative to a fixed coordinate system allows tracing the rise height of a particle after its gets onto a disk surface.

Differential equations of particle motion have been developed and solved using numerical methods. A particle gets onto a disk with specified initial criteria, namely the direction of its entering on a disk and its initial velocity. Two models of particle motion on a disk have been considered. According to the first model, we assume that further particle motion after its getting onto a disk surface is performed due to the kinetic energy of a particle at the moment of its getting onto a disk surface. According to the second model, the backup force of other particles, which breaks the force of particle friction on a surface, is taken into account.

Differential equations of particle motion have been developed in projections onto a fixed solid system of axes. Thus, the system includes three differential equations in three unknown functions. One of the unknown functions is the surface reaction force and the dependences, which describe relative particle motion on a disk surface.

For the purpose of the quality of soil loosening, we conducted multivariate experiment to determine the area of the soil dissipation. On the basis of field experiments, it has been determined that the production process quality of tillage machine meets agrotechnical requirements.
\end{abstract}

KEYWORDS: soil-tilling disk; rotary motion; differential equations of motion.

\section{INTRODUCTION}

Concave disks are widely spread operating elements of tillage machinery. They are mounted at an incidence angle to the direction of a machine movement, which results in a reaction force that rotates disks. Here, soil particles slide on the internal operating surface of a disk, thus, perform relative motion. A grain or material particle performs a compound motion; its part is a relative trajectory of particle sliding on the internal surface of a disk. Investigation of such motion by the example of a separate particle allows understanding the nature of particle movement and determining the influence of the parameters of disk mounting on their behaviour. In its turn, this contributes to designing tillage machinery with disk operating elements. The compound motion of process material particles on running surfaces is considered in fundamental studies [14, 15, 20. Motion of soil grains on a plough mould board is investigated in studies [2, 10]. The determination of the trajectory of a particle, which moves on a cylindrical surface under the action of backup forces, is considered in papers [11, 12, 16]. There is a separate group of scientific papers, which investigate particle motion on a rough surface under the action of a weight force [1, 3, 9, 13, 17, 20].

\section{MATERIAL AND METHOD}

For the purpose of studying patterns of a particle along the inner surface of a spherical cultivating disk that rotates around a horizontal axis, let us consider tillage machinery, presented in Figure 1.

Disk layout in tillage machinery is shown in Figure2 a in projections. A disk is represented by a segment of a sphere of radius $R$, which periphery is a circle of radius $r$ and this circle is a cutting edge, that is to say, a blade. If a machine moves in $Y$-direction, the angle $\alpha$, which is made by a blade surface and $Y$-axis, is an incidence angle. A disk penetrates into the soil at depth $a$. In order to simplify calculations, we do not rotate a disk through angle $\alpha$, as it is shown in 

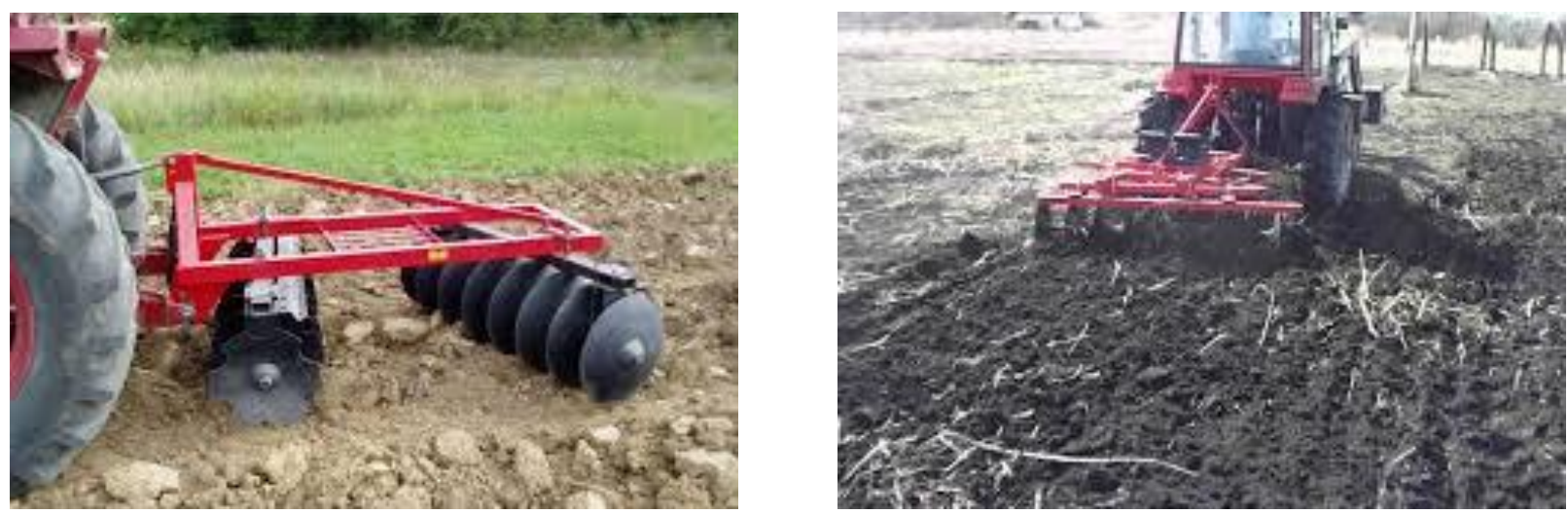

FIGURE 1. Overall view of tillage machinery with a spherical cultivating disk.
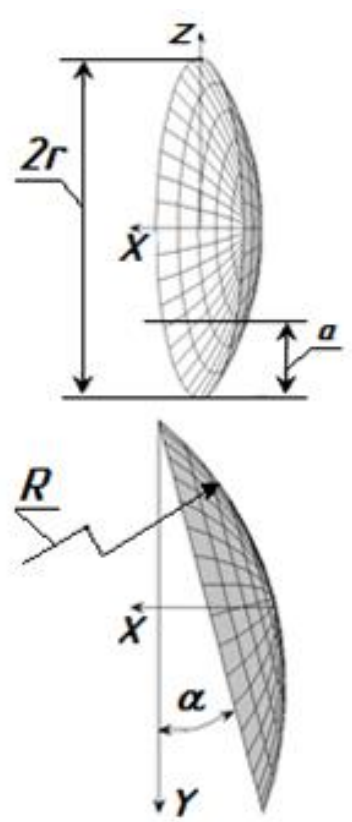

a
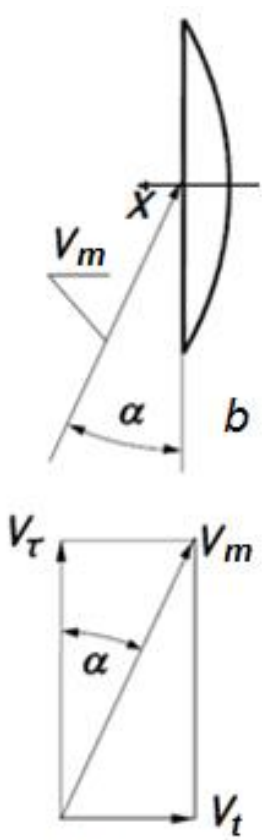

C

FiguRE 2. Illustrations of disk layout in coordinates

Figure 2a, but we assume that the soil approaches a disk at a velocity $V_{m}$ at an angle $\alpha$, where $V_{m}-$ the velocity of machine movement (Figure 2p). Such model enables a simplified description of a disk surface without its rotation trough an angle $\alpha$, by replacing the disk rotation to a rotation of a velocity vector of a machine.

The value of an angular velocity of a disk rotation depends on an incidence angle $\alpha$. At $\alpha=90^{\circ}$, the rotation is not possible. With the decrease of the angle $\alpha$, the angular velocity of disk rotation increases and at $\alpha=0^{\circ}$, it is maximum. In order to determine its value, let us resolve the machine velocity vector $V_{m}$ into two components: longitudinal $V_{\tau}$ and transverse $V_{t}$ (Figure 2b). The value of the components can be found from angle $\alpha$ as $V_{\tau}=V_{m} \cos \alpha, V_{t}=V_{m} \sin \alpha$. A particle gets onto a disk surface having transverse velocity $V_{t}$, and an angular velocity of a disk rotation

depending on the longitudinal velocity $V_{\tau}$. For an effective operation of a machine, the angle $\alpha$ should be increased, but there is a certain limit at which a disk dragging begins. Let us assume that there is no dragging, that is to say, $V_{\tau}=\omega r$, where $\omega-$ angular velocity of the disk rotation. Hence, we define $\omega$ as $\omega=V_{m} \cos \alpha / r$. The value of the transverse velocity $V_{t}$, as well as the angular velocity of the disk rotation, is taken into account for numerical integration of differential equations relative to particle motion on a disk surface.

Let us govern the initial position of a sphere using parametric equations; here $X$-axis of coordinates, which is parallel to a horizontal plane, that is to say, which is parallel to a field surface, is taken for a rotational axis (Figure 2b):

$$
\begin{aligned}
& X=-R \cos u, \\
& Y=R \sin u \sin v, \\
& Z=R \sin u \cos v,
\end{aligned}
$$

where $u, v$ - independent variables of a surface; $v$ - angle of rotation of a point around $X$-axis along the parallel $(v=0-2 \pi) ; u$ - an angular coordinate of this point along the meridian, which origin starts from the point of the intersection of the $X$-axis and a sphere surface.

In order to separate the required segment out of the equations of a sphere (1D), it is necessary to set the limits of the variable $u$. The variable $u$ changes from 0 (corresponds to a segment point, through which axis of rotation passes) to $\arcsin (r / R)$ - this value $u$ corresponds to a segment circle (cutting edge) [9]. If independent variables of a surface $u$ and $v$ are made dependent on one another, a line will be described on the surface of a segment. Let us assume that such dependence is determined by the other variable $t-$ time of the particle sliding on the surface of a segment (a disk). Then, an intrinsic equation relative to particle trajectory is defined by $u=u(t)$ and $v=v(t)$. These dependences are to be found. Equations (1) are the equations of a surface, but when determining dependences $u=u(t)$ and $v=v(t)$ they change into the 
equations of a line on a surface. In order to differentiate equations of a surface from equations of a curve on it, for indicating $X, Y, Z$ in the equations of a surface we use upper case and in the equations of a line we use lower case. With dependences $u=u(t)$ and $v=v(t)$ (yet unknown), equations (1) trace out a trajectory of relative particle motion. Using a differentiation (1) according to time $t$, we obtain projections of a relative velocity of particle sliding on a disk surface:

$$
\begin{aligned}
& x^{\prime}=R u^{\prime} \sin u, \\
& y^{\prime}=R\left(u^{\prime} \cos u \sin v+v^{\prime} \sin u \cos v\right), \\
& z^{\prime}=R\left(u^{\prime} \cos u \cos v-v^{\prime} \sin u \sin v\right) .
\end{aligned}
$$

The value of relative velocity is obtained from vector sum of the components:

$$
V=\sqrt{{x^{\prime}}^{2}+{y^{\prime}}^{2}+{z^{\prime}}^{2}}=R \sqrt{{u^{\prime}}^{2}+{v^{\prime}}^{2} \sin ^{2} u} .
$$

When rotating around its axis at angular velocity $\omega$, all segment points (1) turn at angle $\varphi=-\omega t$. Sign "-" was adopted in order to correlate velocity vector of a machine and rotation direction of a segment (a disk). By applying rotation formulas, let us write parametric equations of a segment, which describe its position after its turn at an angle $\varphi$ :

$$
\begin{aligned}
& X=-R \cos u, \\
& Y=R \sin u \sin v \cos (-\omega t)-R \sin u \cos v \sin (-\omega t), \\
& Z=R \sin u \sin v \sin (-\omega t)+R \sin u \cos v \cos (-\omega t) .
\end{aligned}
$$

After simplifications (4), we obtain an equation of a line on a segment surface, which is an absolute trajectory of particle motion; that is why we pass on to lower case letters:

$$
\begin{aligned}
& x=-R \cos u, \\
& y=R \sin u \sin (v+\omega t), \\
& z=R \sin u \cos (v+\omega t) .
\end{aligned}
$$

The differential equation of particle motion on the surface of a segment of a sphere is written as $m \vec{w}=\vec{F}$, where $m$ - a mass of a particle, $\vec{w}$ - the vector of absolute acceleration, $\vec{F}$ - equal vector forces, which are exerted on a particle. Such forces are the following: weight force of a particle $m g\left(g=9.81 \mathrm{~m} / \mathrm{s}^{2}\right)$, surface reaction $\vec{N}$ and friction force $f \vec{N}$, which offers a resistance to the particle sliding on the surface of a segment ( $f$ - friction coefficient). Let us write the deduced vector equation in projections onto an axis and, as a result, we obtain a set of three differential equations.

Absolute acceleration of a particle is obtained from successive differentiation of equations (5) according to time $t$. The first-order derivative of equations (5), that is to say a vector of an absolute velocity of a particle, takes the following form:

$$
\begin{array}{rl}
x^{\prime}=R & R u^{\prime} \sin u, \\
y^{\prime}=R & \left(u^{\prime} \cos u \sin (v+\omega t)\right. \\
& \left.\quad+\left(v^{\prime}+\omega\right) \sin u \cos (v+\omega t)\right), \\
z^{\prime}=R & \left(u^{\prime} \cos u \cos (v+\omega t)\right. \\
& \left.\quad-\left(v^{\prime}+\omega\right) \sin u \sin (v+\omega t)\right) .
\end{array}
$$

We differentiate equations (6) and obtain projections of a vector of an absolute acceleration onto an axis:

$$
\begin{aligned}
x^{\prime \prime} & =R\left(u^{\prime 2} \cos u+u^{\prime \prime} \sin u\right), \\
y^{\prime \prime} & =R \cos (v+\omega t)\left(2 u^{\prime}\left(v^{\prime}+\omega\right) \cos u+v^{\prime \prime} \sin u\right) \\
& +R \sin (v+\omega t)\left(u^{\prime \prime} \cos u-\left({u^{\prime}}^{2}+\left(v^{\prime}+\omega\right)^{2}\right) \sin u\right), \\
z^{\prime \prime} & =-R \sin (v+\omega t)\left(2 u^{\prime}\left(v^{\prime}+\omega\right) \cos u+v^{\prime \prime} \sin u\right) \\
& +R \cos (v+\omega t)\left(u^{\prime \prime} \cos u-\left(u^{\prime 2}+\left(v^{\prime}+\omega\right)^{2} \sin u\right) .\right.
\end{aligned}
$$

The first exerted force is the particle weight $m g$. Since a vector of weight is down-directed, its projections onto an axis are

$$
\{0 ; 0 ;-m g\} \text {. }
$$

The second exerted force is surface reaction $\vec{N}$ of a segment, which is directed normal to a surface. All the normal lines of a sphere are directed toward its centre, that is why, their coordinates are equal to parametric equations (1), having taken an opposite sign and taking into account surface effect on a particle. This vector is applied to a particle at its locating point. It must be turned through angle $\varphi=-\omega t$, so that it corresponds to the point of its location on the surface. After the turn, projections of a vector take the following:

$$
\begin{aligned}
& N_{x}=\cos u, \\
& N_{y}=-\sin u \sin (v+\omega t), \\
& N_{z}=-\sin u \cos (v+\omega t) .
\end{aligned}
$$

As may be inferred from (9), projections of a vector differ from equations (5) by a sign and the absence of radius $R$, since this is a unit vector.

The last one is the friction force $f \vec{N}$, which is directed opposite to the relative velocity $\vec{V}$ of particle motion, that is to say, at a tangent to a relative trajectory. Projections of a unit vector $\vec{T}$, along which velocity of a particle is directed, are determined by dividing velocity components (2) by its modulus (3) with further rotation through angle $\varphi=-\omega t$ :

$$
\begin{aligned}
& T_{x}=\frac{u^{\prime} \sin u}{\sqrt{{u^{\prime}}^{2}+v^{\prime 2} \sin ^{2} u}}, \\
& T_{y}=\frac{u^{\prime} \cos u \sin (v+\omega t)+v^{\prime} \sin u \cos (v+\omega t)}{\sqrt{{u^{\prime}}^{2}+v^{\prime 2} \sin ^{2} u}}, \\
& T_{z}=\frac{u^{\prime} \cos u \cos (v+\omega t)-v^{\prime} \sin u \sin (v+\omega t)}{\sqrt{{u^{\prime 2}}^{2}+v^{\prime 2} \sin ^{2} u}} .
\end{aligned}
$$


Now, we can write differential equations relative to particle motion taking into consideration the exerted weight force (8), surface reaction $\vec{N}$ and friction force $f \vec{N}$, which are vectored by unit vectors $(9)$ and $(10)$.

The vector equation $m \vec{w}=\vec{F}$ is written in projections on an axis taking into consideration that projections of a vector of an absolute acceleration $\vec{w}$ are represented by expressions (7):

$$
\begin{aligned}
m x^{\prime \prime} & =N \cos u-f N \frac{u^{\prime} \sin u}{\sqrt{{u^{\prime}}^{2}+{v^{\prime}}^{2} \sin ^{2} u}}, \\
m y^{\prime \prime} & =-N \sin u \sin (v+\omega t) \\
& -f N \frac{u^{\prime} \cos u \sin (v+\omega t)+v^{\prime} \sin u \cos (v+\omega t)}{\sqrt{{u^{\prime}}^{2}+v^{\prime 2} \sin ^{2} u}}, \\
m z^{\prime \prime} & =-m g-N \sin u \cos (v+\omega t) \\
& -f N \frac{u^{\prime} \cos u \cos (v+\omega t)-v^{\prime} \sin u \sin (v+\omega t)}{\sqrt{{u^{\prime}}^{2}+v^{\prime 2} \sin ^{2} u}} .
\end{aligned}
$$

Let us substitute, in (11), the expressions of other derivatives from (7) and let us solve the system (11) for other unknown functions $u=u(t)$ and $v=v(t)$, as well as $N=N(t)$. As a result of simplifications we obtain:

$$
\begin{gathered}
u^{\prime \prime}=-f u^{\prime} \frac{R\left(v^{\prime}+\omega\right)^{2} \sin ^{2} u+R u^{\prime 2}-g \sin u \cos (v+\omega t)}{R \sqrt{{u^{\prime 2}}^{2}+{v^{\prime}}^{2} \sin ^{2} u}} \\
+\cos u\left(\left(v^{\prime}+\omega\right)^{2} \sin u-\frac{g}{R} \cos (v+\omega t)\right), \\
v^{\prime \prime}=-f v^{\prime} \frac{R\left(v^{\prime}+\omega\right)^{2} \sin ^{2} u+R u^{\prime 2}-g \sin u \cos (v+\omega t)}{R \sqrt{{u^{\prime}}^{2}+{v^{\prime}}^{2} \sin ^{2} u}} \\
+\frac{g \sin (v+\omega t)}{R \sin u}-\frac{2 u^{\prime}\left(v^{\prime}+\omega\right) \cos u}{\sin u}, \\
N=m\left(R\left(v^{\prime}+\omega\right)^{2} \sin ^{2} u+R u^{\prime 2}\right. \\
-g \sin u \cos (v+\omega t)) .
\end{gathered}
$$

Expressions 12 should be viewed as a system of the first two equations. The third equation - surface reaction - becomes known after solving the system of the first two equations. It must be solved using numerical methods.

Initial conditions of an integration are chosen proceeding from the point on a blade at which a particle gets onto a surface. This point can be chosen within the limits of the soil penetration depth $a$ of a disk and it is specified by initial coordinates $u$ and $v$. At $u=\arcsin (r / R)$ and at $v=\pi$ the point is located in the lowermost part of a disk, that is at depth $a$. At $u=\arcsin (r / R)$ and at $v=\pi-\arccos ((r-a) / r)$ the point is located in such part of a blade, where it meets a field surface. When choosing the parameter $\mathrm{v}$ within the specified limits, we choose a point on a blade at a digging depth of penetration.

The initial value of first-order derivatives $u^{\prime}$ and $v^{\prime}$ determine the direction of the particle entering a surface. At $u=\arccos (r / R)-$ const. and a change of the independent value $v$, a circle — disk periphery - is described on a surface. The derivative $v^{\prime}$ represents the angular velocity of particle sliding at the moment of it getting onto a blade. Since, before entering a disk, a particle is stationary, then, relative to a disk, which rotates with angular velocity being $\omega=V_{m} \cos \alpha / r$, we assume $v^{\prime}=-\omega$. If a disk rotated and a particle remained in place, the velocity of sliding on a blade would be stabilized, opposite to the direction of disk rotation and would be equal to $v^{\prime}=-\omega$. But it happens only at the moment of particle getting onto a blade, after that the particle is trapped by a surface, moves along it and the angular velocity of sliding decreases. Derivative $u^{\prime}$ represents angular velocity of particle motion along a coordinate line (angle) $u$, that is to say, along the meridian, which goes from the point on a blade to the centre of a disk. Let us assume that linear velocity of particle motion in a meridian line, which is determined by expression $R u^{\prime}$, is equal to cross stream velocity component of machine movement, that is $V_{m} \sin \alpha$. Hence, we obtain the initial value of $u^{\prime}$ as $u^{\prime}=V_{m} \sin \alpha / R$.

\section{Results}

When conducting the research, it was assumed that a machine moves at $9 \mathrm{~km} / \mathrm{h}=2.5 \mathrm{~m} / \mathrm{s}$, an incidence angle is $\alpha=20^{\circ}$, design parameters of a disk are $R=0.5 \mathrm{~m}, r=0.225 \mathrm{~m}$, tillage depth is $a=0.08 \mathrm{~m}$.

Using the deduced dependences, we determine initial conditions of the integration $u=0.47 \mathrm{rad} ; v=$ $2.27-\pi$ (depending on the depth, at which a particle enters a disk blade); $u^{\prime}=1.72 \mathrm{~s}^{-1} ; v^{\prime}=-\omega=-10.4 \mathrm{~s}^{-1}$. As a result of a numerical integration of the system (12) relative (following formulas (1)) and absolute (following formulas (5)) trajectories of particle motion have been made. They are shown in Figure 3 from the view point, which direction is perpendicular to a blade surface. Full lines show trajectories of absolute motion of a particle, which gets onto a blade at a digging depth and dashed lines show relative trajectories, that is to say, trajectories of a particle sliding on a disk.

It is to be recalled that we have accepted a geometric model, according to which a disk rotates about a horizontal axis but does not move on the field. Instead, a particle is delivered on it at an incidence at the specified initial velocity $V_{m}$, which falls into two components: longitudinal and transverse. Trajectories of sliding (dashed lines) are not interesting for the investigation, but absolute trajectories (full lines) give us an idea how particles move relative to the disk projection, particularly their rise height.

Figure $3 \mathrm{p}$ illustrates absolute trajectories of particle motion when they get onto a disk at the same penetration depth as we have in the first case, but at an incidence angle being of $\alpha=30^{\circ}$. Initial conditions of the integration are chosen according to this angle at previous velocity of machine movement: $u^{\prime}=2.5 \mathrm{~s}^{-1}$; $v^{\prime}=-\omega=-9.6 \mathrm{~s}^{-1}$. Figure $3 \mathrm{~b}$ shows that the rise 

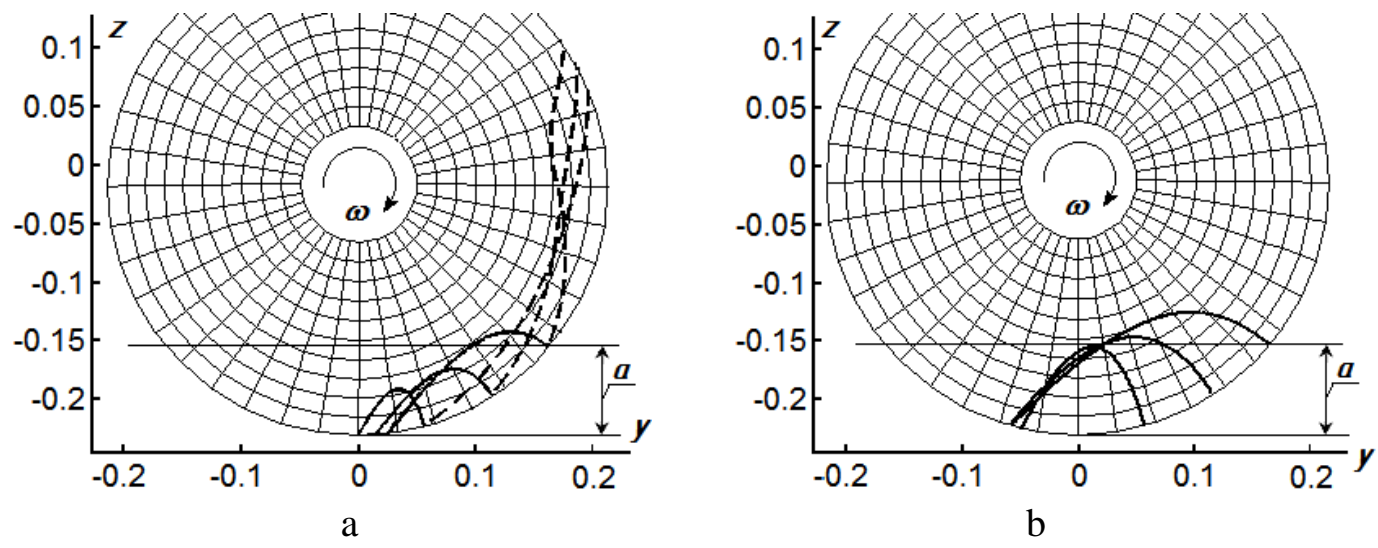

FiguRE 3. Projection of a disk and plotted trajectories of particle motion: a) absolute and relative trajectories at an incidence angle being of $\alpha=20^{\circ}$; b) absolute trajectories at an incidence angle of $\alpha=3^{\circ}$.
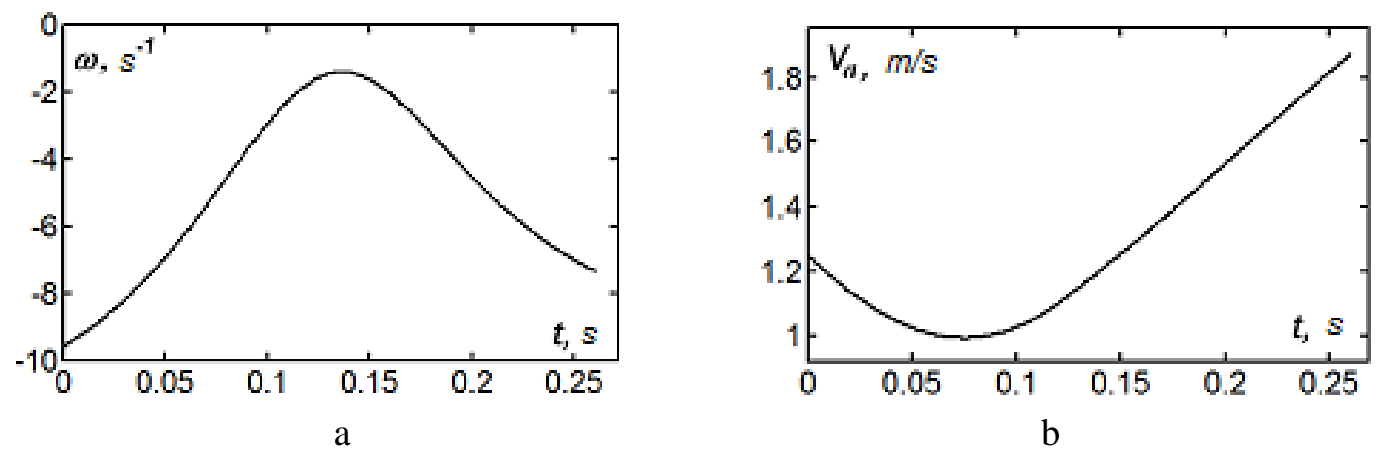

FIGURE 4. Kinematic characteristics of particle motion at $\alpha=30^{\circ}$, when it gets onto a blade at its contact point with a field surface (a peak trajectory in Figure 2 $\mathrm{b}$ ): a) a curve of an angular velocity of the particle sliding; b) a curve of an absolute velocity of motion.

height of particles increases and the area of particle dispersion on a disk surface increases as well.

Figure 4 4 shows a curve of angular sliding velocity of a particle, which gets onto a blade at a point of its contacting a field surface at $\alpha=3^{\circ}$ (a peak trajectory in Figure 3p). From initial value $v^{\prime}=-\omega=-9.6 \mathrm{~s}^{-1}$, the value of angular velocity decreases due to surface friction and then it begins to increase when a particle moves downward. The duration of such movement until it reaches the lowest point is $0.26 \mathrm{~s}$. Figure 4 b shows a curve of an absolute velocity of particle motion.

The initial value of the velocity equals to transverse component $V_{m} \sin \alpha=1.25 \mathrm{~m} / \mathrm{s}$, which corresponds to the initial angular velocity $u^{\prime}=V_{m} \sin \alpha / R=2.5 \mathrm{~s}^{-1}$ at the point of its getting onto the surface. After this, the velocity decreases, which corresponds to the rise of a particle and then it increases again. In all the examples, the coefficient of the particle friction on a disk surface is assumed to be $f=0.3$.

The deduced model of particle motion only vaguely describes the real process. This model assumes that further motion of a particle, after it gets onto a surface, is performed due to the kinetic energy of a particle at the moment of it getting onto a disk surface. Practically, there are backup forces of adjacent particles, which make a particle move on a surface. It can be assumed that this backup force breaks friction resistance force, that is to say, these forces are equilibrated and, in this case, it is possible to adopt a friction coefficient $f$ to be equal to zero. However, this is fair only at the stage of a particle rise and at the stage of its downward motion, the backup force may act as a decelerating force, which conflicts with a real process. Figure 5a shows enlarged trajectories of absolute particle motion for $\alpha=3^{\circ}$ at $f=0.3$ (a full heavy line; the same trajectories in a scaled-down version are illustrated in Figure 3p) and at $f=0$ (a fine dashed line). Having analysed these trajectories, it can be concluded that the value of friction coefficient has a minor influence on the trajectory of the absolute motion. The increase of the coefficient of the soil friction on a disk surface from $f=0$ to $f=0.3$ results in the change of motion trajectories of soil particles at an incidence angle of $\alpha=30^{\circ}$ in case when a disk rotates at angular velocity being $\omega=9.6 \mathrm{~s}^{-1}$ within the range of $3-6 \%$. But the same cannot be stated in relation to a stationary disk, that is at $\omega=0$ (for example, the one that is jammed). Figure $5 \mathrm{p}$ shows the absolute trajectories at the same parameter values but for a stationary disk. The increase of the coefficient of soil friction on a disk surface from $f=0$ to $f=0.3$ results in the change of motion trajectories of soil particles 


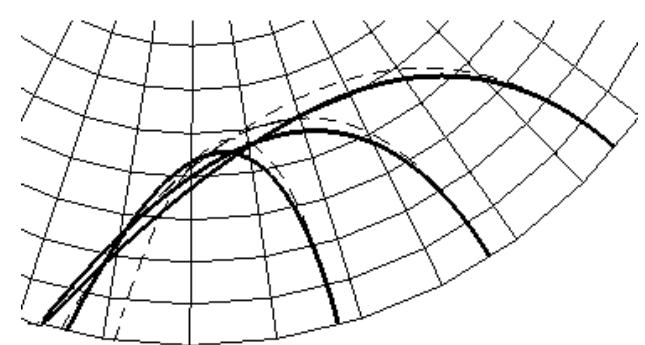

a

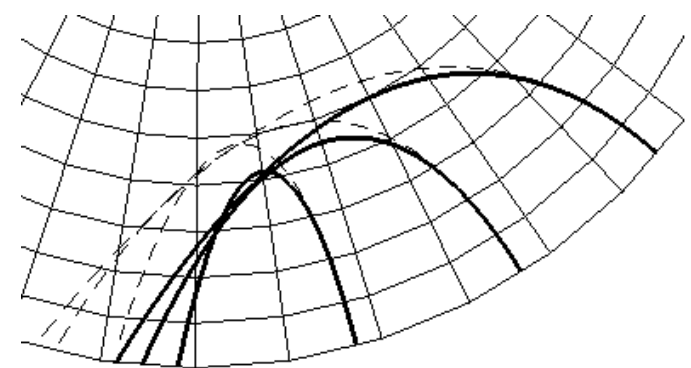

b

FiguRE 5. Disk segments with plotted absolute trajectories of particle motion at an incidence angle of $\alpha=30^{\circ}$ and various friction coefficients (a full line at $f=0.3$ and a dashed line at $f=0$ ): a) a disk rotates at an angular velocity of $\left.\omega=9.6 \mathrm{~s}^{-1} ; \mathrm{b}\right)$ a disk is stationary $(\omega=0)$.

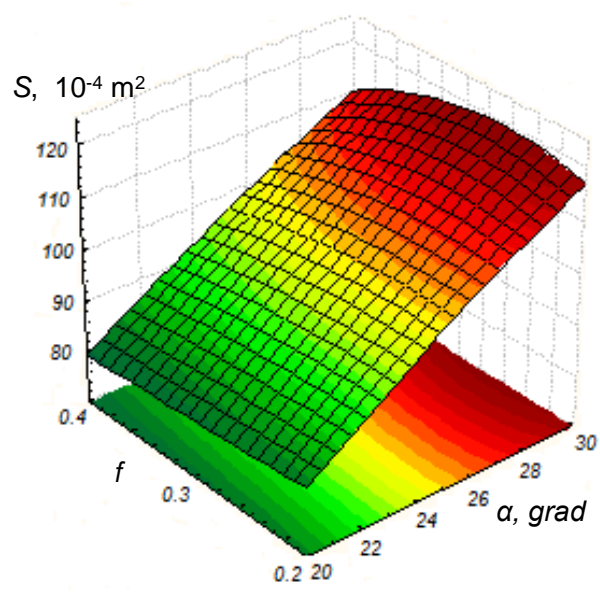

a

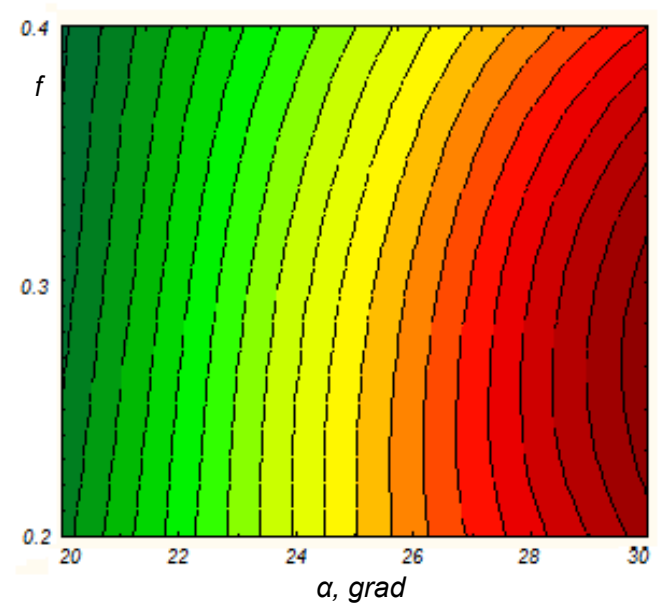

$\mathrm{b}$

Figure 6. The change dependence of the area of particle dispersion $S$ on an incidence angle of a disk $\alpha$ and friction coefficient $f$ as functional $S=F(\alpha, f)$.

at an incidence angle of $\alpha=300^{\circ}$ in case when a disk is jammed within the range of $12-20 \%$.

It is worth mentioning that the trajectories, which are plotted using dashed lines in Figure 5 ab are absolutely the same. It means that when there is no friction, that is to say, a surface is perfectly smooth, an absolute trajectory does not depend on the value of an angular velocity of its rotation. Friction forces influence the trajectories of particle motion on a stationary disk and a moving one. In both cases, the trajectories differ within the range of $4-7 \%$. at the stage of a particle rise and they differ within the range of $12-18 \%$. at the stage of a particle downward movement. This can be explained by the fact that friction forces on a moving disk draw a particle in the direction of its rotation.

The investigation of the kinematic parameters of the movement of soil particles on the inner surface of a disk working body that rotates around a horizontal axis and moves at an angle of attack in relation to the movement of the unit, facilitates the design of the disk tillage tools working bodies.

Also investigation of such motion by the example of a separate particle allows understanding the nature of particle movement and determining the influence of the parameters of disk mounting on their behaviour.

On the basis of field experiments, it has been determined that the production process quality of a tillage machine meets the agrotechnical requirements.

In the process of the experimental studies of a spherical cultivating disk, the following parameters were variable: the angular velocity of disk rotation $\omega=0--10 \mathrm{~s}^{-1}$; the incidence angle of the disk $\alpha=20^{\circ}-30^{\circ}$ and the friction coefficient $f=0.2-0.4$. After processing numerical values, regression equations, which define the area of particle dispersion $S$ according to the change of the above mentioned parameters, have been obtained:

$$
S=9.97+3.46 \alpha+1.76 \omega-4.64 \alpha \omega .
$$

Having analysed the regression equations (13) response surfaces and their two-dimensional sections, which are illustrated in Figures 6, 7 and 8, were constructed.

The analysis of the surfaces reveals, that the area of particle dispersion $S$ changes within the range of 


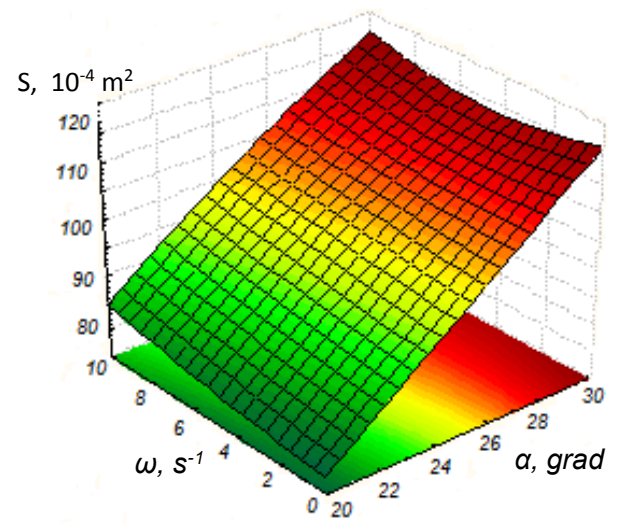

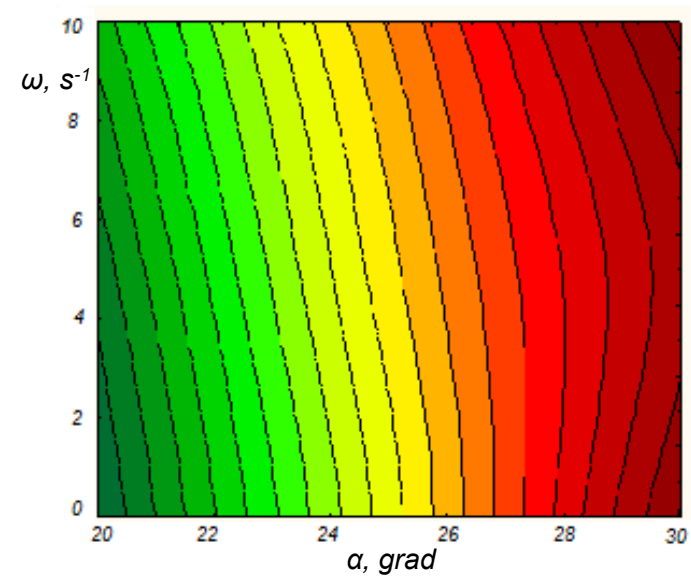

b

FiguRE 7 . The change dependence of the area of particle dispersion $S$ on an incidence angle of a disk $\alpha$ and an angular velocity of the disk rotation $\omega$ as functional $S=F(\alpha, \omega)$.

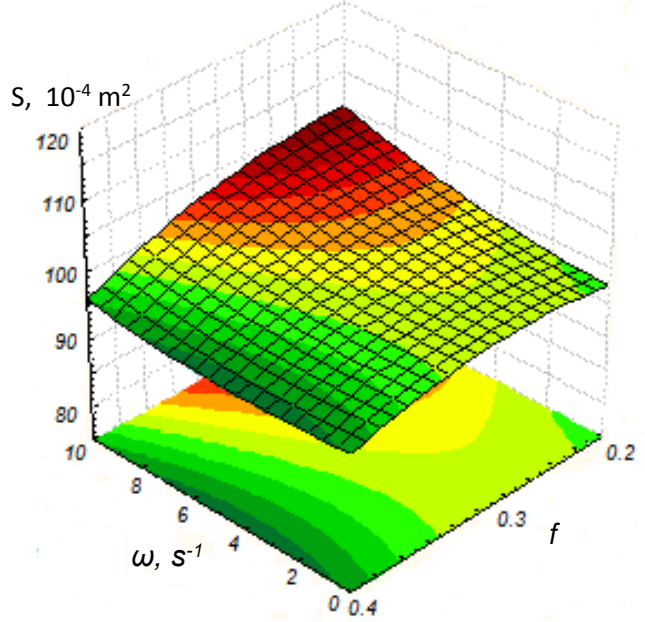

a

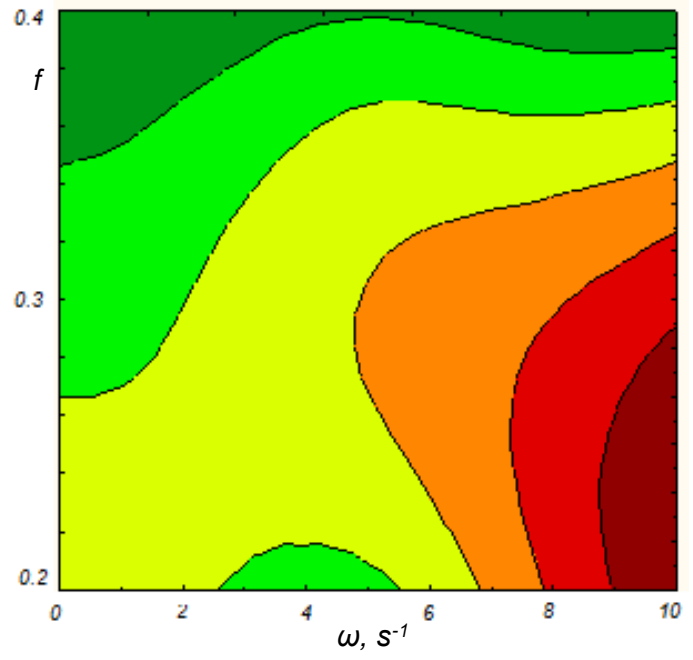

$\mathrm{b}$

FigurE 8. Change dependence of the area of particle dispersion $S$ on an angular velocity of disk rotation $\omega$ and friction coefficient $f$ as functional $S=F(\omega, f)$.

$70 \cdot 10^{-4}-130 \cdot 10^{-4} \mathrm{~m}^{2}$ depending on the change in design and kinematic parameters and process variables of a screw and on loose material characteristics within the following limits: on an incidence angle of a disk $\alpha=20^{\circ}-30^{\circ}$; an angular velocity of the disk rotation $\omega=0--10 \mathrm{~s}^{-1}$; the friction coefficient $f=0.2-0.4$. It has been determined that when an incidence angle of a disk $\alpha$ increases, the area of particle dispersion $S$ increases as well 1.81 times; when an angular velocity of a disk rotation $\omega$ increases, $S$ decreases 1.36 times; within the range of the change in the friction coefficient $f$, the change in $S$ is insignificant.

\section{Conclusion}

A mathematical model of soil particle motion on a disk operating element, which rotates around a fixed horizontal axis, has been developed. The results of the numerical solution of the equations have shown that when the incidence angle increases, the rise height of particles and the area of the particle dispersion on a disk surface increase as well. The duration of such movement until it reaches the lowest point is $0.26 \mathrm{~s}$. The initial value of the velocity equals to transverse component $V_{m} \sin \alpha=1.25 \mathrm{~m} / \mathrm{s}$, which corresponds to the initial angular velocity $u^{\prime}=V_{m} \sin \alpha / R=2.5 \mathrm{~s}^{-1}$ at the point of it getting onto the surface. After this, the velocity decreases, which corresponds to the rise of a particle and then it increases again. The friction coefficient does not significantly influence the trajectory of particle motion. However, if a disk is stationary, it contributes more to the formation of a trajectory of particle motion. When there is no friction, an absolute trajectory does not depend on the value of the angular velocity of its rotation. Friction forces influence the trajectories of particle motion on a stationary disk and a moving one. In both 
cases, the trajectories are the same at the stage of the particle rise and they differ at the stage of the particle downward movement. This can be explained by the fact that friction forces on a moving disk draw a particle in the direction of its rotation.

For the purpose of the quality of soil loosening, we conducted a multivariate experiment to determine the area of the soil dissipation. On the basis of field experiments it has been determined that the production process quality of a tillage machine meets the agrotechnical requirements. The analysis of the surfaces reveals, that the area of particle dispersion $S$ changes within the range of $70 \cdot 10^{-4}-130 \cdot 10^{-4} \mathrm{~m}^{2}$ depending on the change in design and kinematic parameters and process variables of a screw and on loose material characteristics within the following limits: on an incidence angle of a disk $\alpha=20^{\circ}-30^{\circ}$; an angular velocity of the disk rotation $\omega=0-10 \mathrm{~s}^{-1}$; the friction coefficient $f=0.2-0.4$. It has been determined that when an incidence angle of a disk $\alpha$ increases, the area of particle dispersion $S$ increases as well 1.81 times; when an angular velocity of a disk rotation $\omega$ increases, $S$ decreases 1.36 times; within the range of the change in the friction coefficient $f$, the change in $S$ is insignificant.

\section{REFERENCES}

[1] Gevko R.B., Klendiy O.M. (2014) - The investigation of the process of the actuation of the safety device of a screw conveyer, INMATEH: Agricultural engineering, vol. 42, no. 1, pg. 55-60, Bucharest, Romania.

[2] Gyachev L.V. (1961) Theory of plow-bottom surface. Zelenograd, 317 p.

[3] Hevko R.B., Dzyura V.O., Romanovsky R.M., (2014), Mathematical model of the pneumatic-screw conveyor screw mechanism operation, INMATEH: Agricultural engineering, vol.44, no.3, pg.103-110, Bucharest/Romania;

[4] Hevko R.B., Klendii M.B., Klendii O.M. (2016) Investigation of a transfer branch of a flexible screw conveyer, INMATEH: Agricultural engineering, vol. 48, no. 1/2016, pg. 29-34 Bucharest, Romania

[5] Hevko R.B., Rozum R.I., Klendii O.M. (2016) Development of design and investigation of operation processes of loading pipes of screw conveyors, INMATEH: Agricultural engineering, vol.50, no.3, pg.89-96, Bucharest/Romania;

[6] Hevko R.B., Shynkaryk M.I., Vozniuk S.V. (2012) Screw Conveyer, Patent № 68113 Ukraine, MPK B65G $33 / 14$;

[7] Hevko R.B., Yazlyuk B.O., Liubin M.V., Tokarchuk O.A., Klendii O.M., Pankiv V.R. (2017) - Feasibility study of the process of transpotration and stirring of mixture in continuous-flow conveyers, INMATEH: Agricultural engineering, vol.51, no.3, p.g. 49-58 , Bucharest/Romania;

[8] Hevko R.B., Zalutskyi S.Z., Tkachenko I.G., Klendiy O.M. (2015) - Development and investigation of reciprocating screw with flexible helical surface, INMATEH: Agricultural engineering, vol. 46, no.2, pg. 133-138.
[9] Klendii M.B., Klendii O.M. (2016) Inverrelation between incidence ange and roll ange of concave disks of soil tillage implements, INMATEH: Agricultural Engineering, vol.49, no.2, pp.13-20, Bucharest;

[10] Klendii M.B. and Pylypaka S.F. (2016) Analytical model of arranging soil-tilling concave disks for determination of geometrical and technical data // Scientific Bulletin of National University of Life and Environmental Sciences of Ukraine. - Kyiv., Vol. 241. P. $140-150$.

[11] Pylypaka S.F., Klendii M.B., Klendii O.M. (2017) Particle motion over the surface of a rotary vertical axis helicoid, INMATEH: Agricultural engineering, vol.51, no.3, pg. 15-28, Bucharest/Romania;

[12] Shvedik M. (2014) - Analysis of work process of seeding furrows embedding by a conical roller, INMATEH: Agricultural engineering, vol.44, no.3, pg.27-32, Bucharest/Romania;

[13] Sysoev N.I. and Pylypaka S.F. (1949) Theoretical framework and calculation for a sorter "Zmeika" // Selhozmashina [Agricultural Machinery]. - № 8,- P. 5 - 8 .

[14] Vasylenko P.M. (1960) Theory of particle motion on rough surfaces of agricultural machines, Kyiv.:

UASKhN, 1960. - 283 p.

[15] Vasylkovska K.V., Leshchenko S.M., Vasylkovskyi O.M., Petrenko D.I. (2016) - Improvement of equipment for basic tillage and sowing as initial stage of harvest forecasting, INMATEH: Agricultural engineering, vol.50, no.3, pg.13-20, Bucharest/Romania;

[16] Voitiuk D.H. (1999) Revisiting the determination of motion trajectories of soil particles on cylindrical surfaces of operating element of tillage machinery // Collection of Scientific Papers of National Agrarian University "Mehanizatsiia Silskoho Hospodarstva [Mechanization of Agricultural Production]". - Vol 5. "Suchasni Problemy Mehanizatsii Silskoho Hospodarstva [Current Problems of Mechanization of Agriculture]". Kyiv.: NAU, P. 242-251.

[17] Voitiuk D.H. (2002) Determination of motion trajectory of a material particle on gravitational linear surfaces with horizontal generators // Collection of Scientific Papers of National Agrarian University "Mehanizatsiia Silskoho Hospodarstva [Mechanization of Agricultural Production]". - Vol.12. -Kyiv NAU, P.58-69.

[18] Voitiuk D.H. and Pylypaka S.F. (2003)

Determination of motion trajectory of a material particle on a gravitational surface by the example of a developable helicoid // Mehanizatsiia i Enerhetyka Silskoho Hospodarstva [Mechanization and Power Engineering of Agriculture]. IV International Scientific and Technical Conference MOTROL-2003. -Kyiv NAU, Vol 6. - P. 113-126.

[19] Voitiuk D.H. and Pylypaka S.F. (2003) Peculiarities of material particle motion on gravitational linear surfaces // Bulletin of Kharvik State Technical University of Agriculture. - Vol. 21. "Mehanizatsiia Silskoho Hospodarstva [Mechanization of Agriculture]". - Kharkiv, P. 75-88.

[20] Zaika P.M. (1992) Selected problems of agricultural mechanics, Kyiv: USKhA, 507 p. 